\title{
Three-Dimensional Printed Target Plates for Matrix-Assisted Laser Desorption/Ionization Mass Spectrometry
}

Jana Fialova ${ }^{1,2}$, Jaroslav Hrabak ${ }^{3}$, Vendula Studentova ${ }^{3}$, Daniel Kavan ${ }^{1}$, Petr Pompach ${ }^{1,4}$ and Petr Novak ${ }^{1}$.

${ }^{1}$ Institute of Microbiology of the Czech Academy of Sciences, BIOCEV, Prumyslova 595, 25250 Vestec, Czech Republic

${ }^{2}$ Department of Analytical Chemistry, University of Chemistry and Technology Prague, Technicka 5, 16628 Prague 6, Czech Republic

${ }^{3}$ Biomedical Center and Department of Microbiology, Faculty of Medicine in Pilsen, Charles University, alej Svobody 76, 323 00 Plzen, Czech Republic

${ }^{4}$ Institute of Biotechnology of the Czech Academy of Sciences, BIOCEV, Prumyslova 595, 25250 Vestec, Czech Republic

\section{Corresponding Author}

* Petr Novak, Institute of Microbiology of the Czech Academy of Sciences, BIOCEV, Prumyslova 595, 25250 Vestec, Czech Republic, pnovak@biomed.cas.cz 


\section{Comparison of prices of 3D printed and commercial MBT BioTarget}

\section{Equations for mass spectra parameters that were calculated}

\section{List of Equations}

Equation S1.S/N ratio

Equation S2. Limit of detection

Equation S3. Limit of quantification

\section{List for Tables}

Table S1. Detailed calculation of the Limit of detection and limit of quantification for three different targets (Stainless-steel, 3D printed target and ITO slide glass) and comparison at the lowest measured BSA protein concentrations.

Table S2. Comparison of electrical resistivity for MALDI targets used in this article

Table S3. Complete results of six bacteria strains by 3D printed target and MBT BioTarget. SD represents Standard Deviation and RSD Relative Standard Deviation.

\section{List for Figures}

Figure S1. The Schematic draw of 3D printed MALDI target

Figure S2. Comparison of mass spectra for SA matrix and a plane surface on three different targets (Stainless-steel, 3D printed target and ITO slide glass) measured by MALDI-TOF MS.

Figure S3. Comparison of mass resolution for BSA protein using three different targets (Stainless-steel, 3D printed target and ITO slide glass) measured by MALDI-TOF MS.

Figure S4. Comparison of mass spectra for HCCA matrix and a plane surface on two different targets (Stainless-steel and 3D printed target) measured by MALDI-TOF MS. 


\section{Comparison of prices of 3D printed and commercial MBT BioTarget}

\section{Bruker MBT BioTarget}

Bruker MBT BioTarget 96 is on offer for $\$ 1212$. The box contains 20 single pieces of MALDI plates, which means that one MALDI MBT BioTarget 96 plate costs $\$ 60$ and one spot on the MBT BioTarget 96 costs $\$ 63$.

\section{D printing of MALDI targets; 3D printer TRILAB DeltiQL + TRILAB FlexPrint}

The printing time of one MALDI target is about 18 minutes. Theoretically, the printer can print 80 MALDI targets per day. Due to the price of the printer and its amortization, the price of 3D printing of one 96-well target will be $₫ 3$ and price for one single spot $₫ 0.06$. The price of the filament per one target is $\$ 9.2$ and for one single spot is $₫ 0.2$. The price of electricity per one target is $₫ 0.65$ and for one single spot is $\$ 0.01$ in the current situation of the energy market $(07 / 2020)$.

\section{Final price}

FinalPrice $_{\text {target }}=$ PPrice $_{\text {target }}$ FPrice $_{\text {target }}$ EPrice $_{\text {target }}=3.0+9.2+0.65=₫ 13$

FinalPrice $_{\text {spot }}=$ PPrice $_{\text {spot }}$ FPrice $_{\text {spot }}$ EPrice $_{\text {spot }}=0.06+0.2+0.01=\$ 0.3$ 
Equations for mass spectra parameters that were calculated

$\mathrm{S} / \mathrm{N}$ ratio equation:

$\mathrm{S} / \mathrm{N}=\frac{\frac{1}{\mathrm{n}} \sum_{\mathrm{i}=0}^{\mathrm{n}} \mathrm{Y}_{\text {signal }}}{\frac{1}{\mathrm{n}} \sum_{\mathrm{i}=0}^{\mathrm{n}} \mathrm{Y}_{\text {noise }}}$

Equation S1

LOD calculation

The limit of detection is calculated by equation:

$\mathrm{LOD}=Y_{\text {blank }}+3 s_{\text {blank }}[$ signal Intensity $]$

Equation S2

LOQ calculation

The limit of quantification is calculated by equation:

$\mathrm{LOQ}=Y_{\text {blank }}+10 s_{\text {blank }}[$ signal Intensity $]$

Equation S3 
Table S1. Detailed calculation of the Limit of detection and limit of quantification for three different targets (Stainless-steel, 3D printed target and ITO slide glass) and comparison at the lowest measured BSA protein concentrations.

\begin{tabular}{|c|c|c|c|}
\hline Parameter & Stainless-steel target & 3D printed target & ITO glass slide \\
\hline $\mathrm{Y}_{\text {blank }}$ & 33.2 & 29.4 & 5.3 \\
\hline $3 \mathrm{~s}_{\text {blank }}$ & 21.9 & 38.1 & 3.5 \\
\hline 10 blank & 73.0 & 127.1 & 11.7 \\
\hline LOD $_{\text {Intensity }}$ & 55.1 & 67.5 & 8.8 \\
\hline LOQ $_{\text {Instensity }}$ & 106.2 & 156.5 & 17.0 \\
\hline Intensity $\left(\mathrm{c}=0.5 \mu \mathrm{mol} \cdot \mathrm{I}^{-1}\right)$ & 1668 & 4156 & 1381 \\
\hline Intensity $\left(\mathrm{c}=0.25 \mu \mathrm{mol} \cdot \mathrm{I}^{-1}\right)$ & 132.0 & 1286.7 & 55.0 \\
\hline Intensity $\left(\mathrm{c}=0.125 \mu \mathrm{mol} \cdot \mathrm{l}^{-1}\right)$ & Not observed & 83.3 & 8.0 \\
\hline
\end{tabular}


Table S2. Comparison of electrical resistivity for different MALDI targets.

\begin{tabular}{|l|l|}
\hline Type of target & Electrical resistivity $[\Omega \cdot \mathrm{cm}]$ \\
\hline Stainless steel target & 0.1 \\
\hline ITO glass slide & 370 \\
\hline 3D printed target & 35000 \\
\hline
\end{tabular}


Table S3. Complete results of six bacteria strains by 3D printed target and MBT BioTarget. SD represents Standard Deviation and RSD Relative Standard Deviation.

\begin{tabular}{|c|c|c|c|c|c|c|c|c|}
\hline \multirow{2}{*}{ Bacteria } & \multicolumn{4}{|c|}{ MBT BioTarget } & \multicolumn{4}{|c|}{ 3D printed target } \\
\hline & Score & Average & $\mathrm{SD}$ & RSD & Score & Average & $\mathrm{SD}$ & RSD \\
\hline \multirow{7}{*}{ BTS - standard } & 2.25 & \multirow{7}{*}{2.16} & \multirow{7}{*}{0.05} & \multirow{7}{*}{$2.1 \%$} & 2.22 & \multirow{4}{*}{2.220} & \multirow{4}{*}{0.008} & \multirow{4}{*}{$0.37 \%$} \\
\hline & 2.14 & & & & 2.23 & & & \\
\hline & 2.14 & & & & 2.21 & & & \\
\hline & 2.13 & & & & 2.22 & & & \\
\hline & 2.19 & & & & & & & \\
\hline & 2.12 & & & & & & & \\
\hline & 2.14 & & & & & & & \\
\hline \multirow{10}{*}{ Escherichia coli 4225} & 2.33 & \multirow{10}{*}{2.38} & \multirow{10}{*}{0.04} & \multirow{10}{*}{$1.6 \%$} & 2.34 & \multirow{10}{*}{2.31} & \multirow{10}{*}{0.06} & \multirow{10}{*}{$2.5 \%$} \\
\hline & 2.37 & & & & 2.45 & & & \\
\hline & 2.34 & & & & 2.31 & & & \\
\hline & 2.39 & & & & 2.3 & & & \\
\hline & 2.46 & & & & 2.32 & & & \\
\hline & 2.4 & & & & 2.33 & & & \\
\hline & 2.38 & & & & 2.3 & & & \\
\hline & 2.36 & & & & 2.31 & & & \\
\hline & 2.35 & & & & 2.24 & & & \\
\hline & 2.37 & & & & 2.24 & & & \\
\hline \multirow{9}{*}{$\begin{array}{l}\text { Klebsiella pneumoniae } \\
700603\end{array}$} & 2.29 & \multirow{9}{*}{2.31} & \multirow{9}{*}{0.02} & \multirow{9}{*}{$1.0 \%$} & 2.26 & \multirow{9}{*}{2.2} & \multirow{9}{*}{0.2} & \multirow{9}{*}{$7.9 \%$} \\
\hline & 2.31 & & & & 1.76 & & & \\
\hline & 2.26 & & & & 2.28 & & & \\
\hline & 2.3 & & & & 2.34 & & & \\
\hline & 2.32 & & & & 2.32 & & & \\
\hline & 2.28 & & & & 2.28 & & & \\
\hline & 2.33 & & & & 2.25 & & & \\
\hline & 2.32 & & & & 2.26 & & & \\
\hline & 2.33 & & & & 2.27 & & & \\
\hline \multirow{9}{*}{$\begin{array}{c}\text { Pseudomonas } \\
\text { aeruginosa } 3655\end{array}$} & 2.42 & \multirow{9}{*}{2.39} & & & 2.27 & & & \\
\hline & 2.4 & & & & 2.27 & & & \\
\hline & 2.37 & & & & 2.08 & & & \\
\hline & 2.46 & & & & 1.8 & & & \\
\hline & 2.37 & & 0.04 & $1.8 \%$ & 2.18 & 2.2 & 0.2 & $7.6 \%$ \\
\hline & 2.32 & & & & 2.4 & & & \\
\hline & 2.4 & & & & 2.34 & & & \\
\hline & 2.36 & & & & 2.16 & & & \\
\hline & 2.46 & & & & 2.21 & & & \\
\hline
\end{tabular}




\begin{tabular}{|c|c|c|c|c|c|c|c|c|}
\hline & 2.38 & & & & 2.11 & & & \\
\hline \multirow{10}{*}{$\begin{array}{c}\text { Haemophilus influenzae } \\
4457\end{array}$} & 2.53 & \multirow{10}{*}{2.52} & \multirow{10}{*}{0.04} & \multirow{10}{*}{$1.7 \%$} & 2.21 & \multirow{9}{*}{2.2} & \multirow{9}{*}{0.2} & \multirow{9}{*}{$7.1 \%$} \\
\hline & 2.52 & & & & 2.07 & & & \\
\hline & 2.61 & & & & 2.36 & & & \\
\hline & 2.48 & & & & 2.33 & & & \\
\hline & 2.55 & & & & 2.29 & & & \\
\hline & 2.56 & & & & 2.32 & & & \\
\hline & 2.5 & & & & 2.11 & & & \\
\hline & 2.53 & & & & 2.3 & & & \\
\hline & 2.49 & & & & 1.89 & & & \\
\hline & 2.47 & & & & & & & \\
\hline \multirow{10}{*}{$\begin{array}{c}\text { Staphylococcus aureus } \\
4223\end{array}$} & 2.48 & \multirow{10}{*}{2.48} & \multirow{10}{*}{0.03} & \multirow{10}{*}{$1.0 \%$} & 2.35 & \multirow{10}{*}{2.34} & \multirow{10}{*}{0.07} & \multirow{10}{*}{$2.9 \%$} \\
\hline & 2.47 & & & & 2.22 & & & \\
\hline & 2.48 & & & & 2.22 & & & \\
\hline & 2.45 & & & & 2.36 & & & \\
\hline & 2.51 & & & & 2.38 & & & \\
\hline & 2.45 & & & & 2.39 & & & \\
\hline & 2.51 & & & & 2.33 & & & \\
\hline & 2.51 & & & & 2.4 & & & \\
\hline & 2.5 & & & & 2.4 & & & \\
\hline & 2.45 & & & & 2.35 & & & \\
\hline \multirow{10}{*}{$\begin{array}{l}\text { Enterococcus faecalis } \\
4224\end{array}$} & 2.51 & \multirow{10}{*}{2.48} & \multirow{10}{*}{0.03} & \multirow{10}{*}{$1.3 \%$} & 2.21 & \multirow{7}{*}{2.1} & \multirow{7}{*}{0.2} & \multirow{7}{*}{$10 \%$} \\
\hline & 2.41 & & & & 2.45 & & & \\
\hline & 2.49 & & & & 2.32 & & & \\
\hline & 2.47 & & & & 1.96 & & & \\
\hline & 2.49 & & & & 2.27 & & & \\
\hline & 2.51 & & & & 1.92 & & & \\
\hline & 2.5 & & & & 1.89 & & & \\
\hline & 2.44 & & & & & & & \\
\hline & 2.48 & & & & & & & \\
\hline & 2.48 & & & & & & & \\
\hline
\end{tabular}




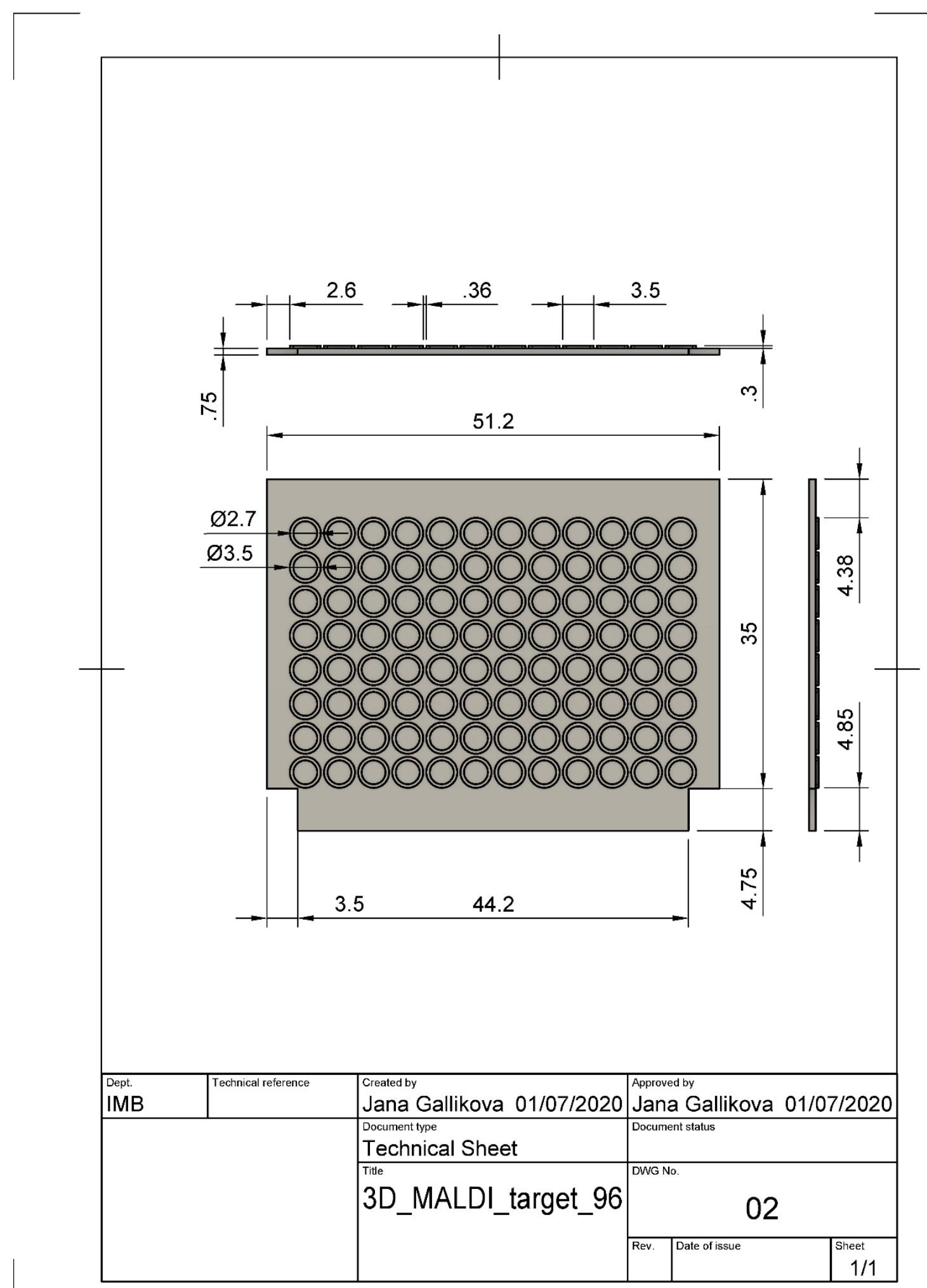

Figure S1. The Schematic draw of 3D printed target. 


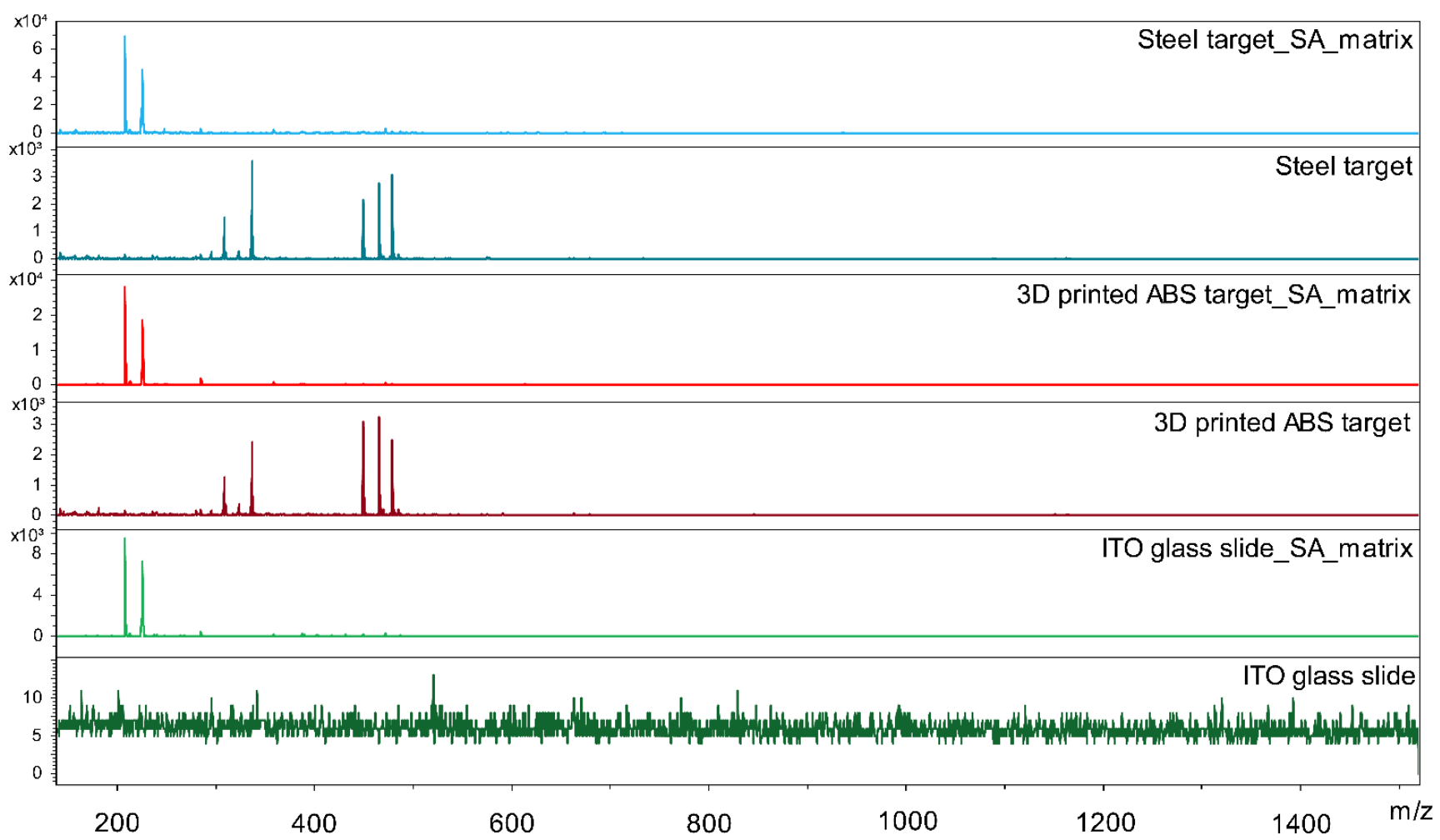

Figure S2. Comparison of mass spectra for SA matrix and a plane surface on three different targets (Stainless-steel, 3D printed target and ITO slide glass) measured by MALDI-TOF MS. 


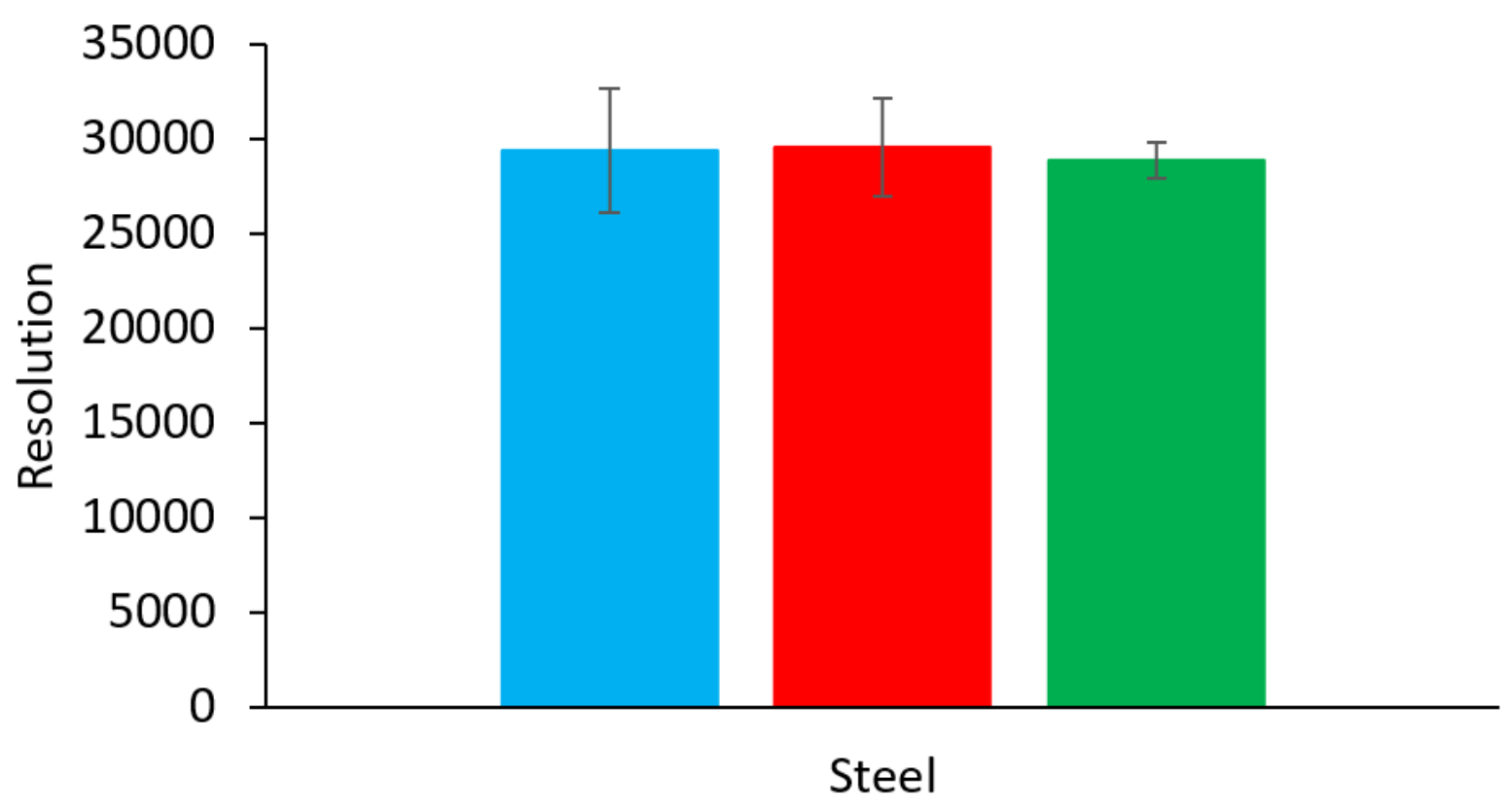

\section{Steel $\square$ ABS $\square$ ITO}

Figure S3. Comparison of mass resolution for BSA protein using three different targets (Stainless-steel, 3D printed target and ITO slide glass) measured by MALDI-TOF MS. 


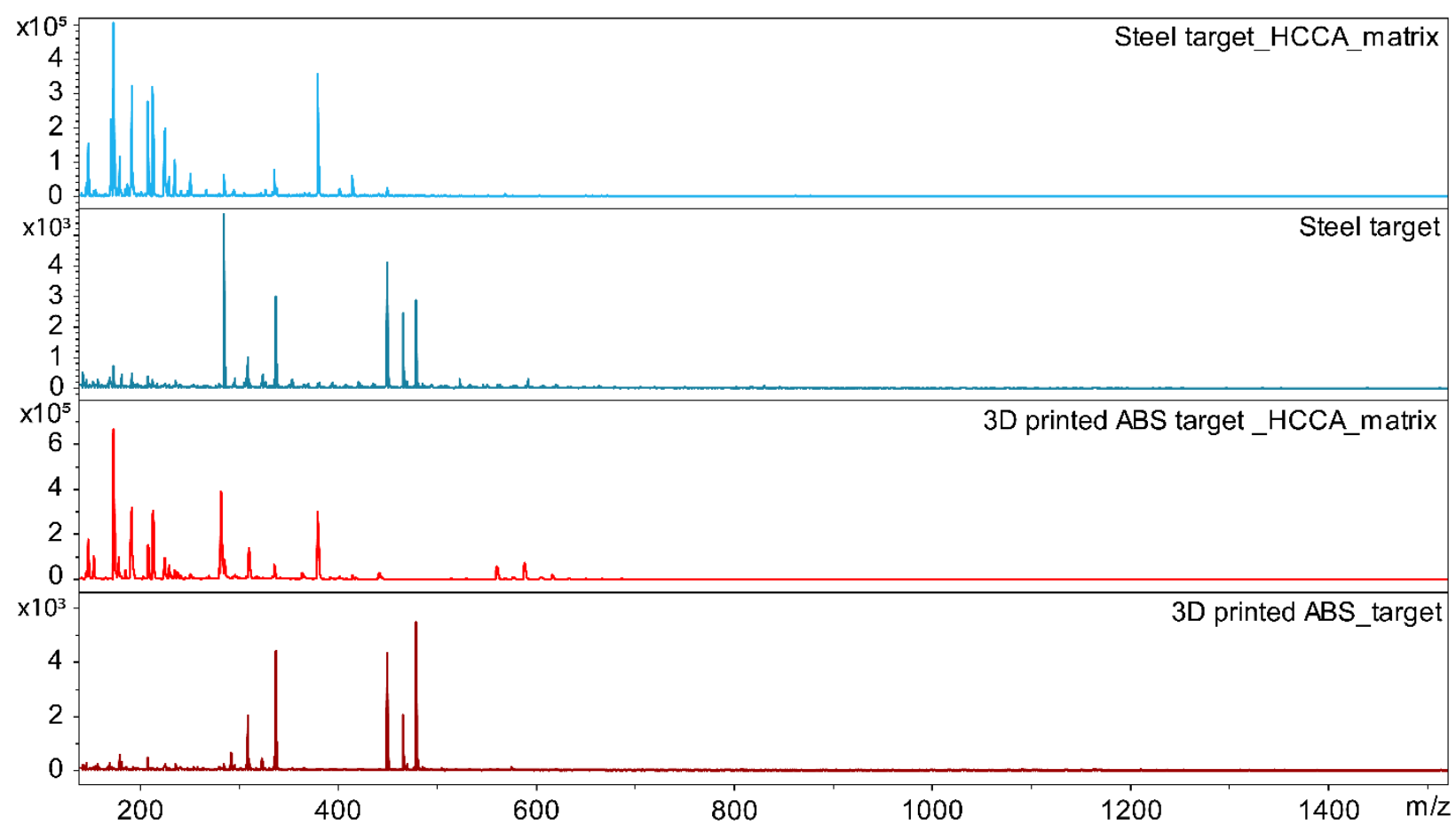

Figure S4. Comparison of mass spectra for HCCA matrix and a plane surface on two different targets (Stainless-steel and 3D printed target) measured by MALDI-TOF MS. 